\title{
Multilinear Square Functions with Kernels of Dini's Type
}

\author{
Zengyan $\mathrm{Si}^{1}$ and Qingying $\mathrm{Xue}^{2}$ \\ ${ }^{1}$ School of Mathematics and Information Science, Henan Polytechnic University, Jiaozuo 454000, China \\ ${ }^{2}$ School of Mathematical Sciences, Beijing Normal University, Laboratory of Mathematics and Complex Systems, Ministry of Education, \\ Beijing 100875, China
}

Correspondence should be addressed to Zengyan Si; zengyan@hpu.edu.cn

Received 9 December 2015; Accepted 31 January 2016

Academic Editor: Rodolfo H. Torres

Copyright (c) 2016 Z. Si and Q. Xue. This is an open access article distributed under the Creative Commons Attribution License, which permits unrestricted use, distribution, and reproduction in any medium, provided the original work is properly cited.

Let $T$ be a multilinear square function with a kernel satisfying Dini(1) condition and let $T^{*}$ be the corresponding multilinear maximal square function. In this paper, first, we showed that $T$ is bounded from $L^{1} \times \cdots \times L^{1}$ to $L^{1 / m, \infty}$. Secondly, we obtained that if each $p_{i}>1$, then $T$ and $T^{*}$ are bounded from $L^{p_{1}}\left(\omega_{1}\right) \times \cdots \times L^{p_{m}}\left(\omega_{m}\right)$ to $L^{p}\left(\nu_{\vec{\omega}}\right)$ and if there is $p_{i}=1$, then $T$ and $T^{*}$ are bounded from $L^{p_{1}}\left(\omega_{1}\right) \times \cdots \times L^{p_{m}}\left(\omega_{m}\right)$ to $L^{p, \infty}\left(\nu_{\vec{\omega}}\right)$, where $\nu_{\vec{\omega}}=\prod_{i=1}^{m} \omega_{i}^{p / p_{i}}$. Furthermore, we established the weighted strong and weak type boundedness for $T$ and $T^{*}$ on weighted Morrey type spaces, respectively.

\section{Introduction and Main Results}

Let $m(t) \in L^{\infty}$ and $a \in$ BMO. In 1978, Coifman and Meyer [1] introduced a class of multilinear operators as a multilinearization of Littlewood-Paley $g$-function as follows:

$$
\mathscr{B}(a, f)=\int_{0}^{\infty}\left(f * \phi_{t}\right)\left(a * \Phi_{t}\right) \frac{m(t)}{t} d t,
$$

where $\widehat{\phi}$ and $\widehat{\Phi}$ have compact support with $0 \notin$ supp $\widehat{\Phi}$. They studied the $L^{2}$ estimate of $\mathscr{B}$ by using the notion of Carleson measures. In 1982, letting $m \geq 2$ and $p \geq 1$, Yabuta [2] obtained the $L^{p}$ boundedness and BMO type estimates of $\mathscr{B}$ by weakening the assumptions in [1]. In 2002, Sato and Yabuta [3] studied the $\left(L^{p_{1}} \times \cdots \times L^{p_{m}}, L^{p}\right)$ boundedness of the following multilinear Littlewood-Paley $g$-function:

$$
T_{g}(\vec{f})(x)=\int_{0}^{\infty} \prod_{i=1}^{m}\left(\left(\phi_{i}\right)_{t} * f\right)(x) \frac{d t}{t} .
$$

The kernels in (1) and (2) are restricted to separable variable kernels. Thus, efforts have been made to study the above operators with kernels of nonseparated type. In 2015, Xue et al. [4] introduced and studied the weighted estimates for the following multilinear Littlewood-Paley $g$-function with convolution type kernel:

$$
g(\vec{f})(x)=\left(\int_{0}^{\infty}\left|\psi_{t} * \vec{f}(x)\right|^{2} \frac{d t}{t}\right)^{1 / 2},
$$

where

$$
\psi_{t} * \vec{f}(x)=\int_{\left(\mathbb{R}^{n}\right)^{m}} \psi_{t}\left(y_{1}, \ldots, y_{m}\right) \prod_{j=1}^{m} f_{j}\left(x-y_{j}\right) d y_{j},
$$

with $\psi_{t}\left(y_{1}, \ldots, y_{m}\right)=\left(1 / t^{m n}\right) \psi\left(y_{1} / t, \ldots, y_{m} / t\right)$.

The importance of multilinear Littlewood-Paley $g$ function and related multilinear Littlewood-Paley type estimates were shown in PDE and other fields (see [5-10]). For other recent works about multilinear Littlewood-Paley type operators, see [11,12] and the references therein.

In this paper, our aim is to study the boundedness of multilinear square functions with more rough kernels. To begin with, we give some notations and introduce some definitions. The following multiple weights classes $A_{\vec{p}}$ were introduced and studied by Lerner et al. [13]. 
Definition 1 ( $A_{\vec{p}}$ weights class [13]). Let $1 \leq p_{1}, \ldots, p_{m}<\infty$, $1 / p=1 / p_{1}+\cdots+1 / p_{m}$. Given $\vec{\omega}=\left(\omega_{1}, \ldots, \omega_{m}\right)$, set $\nu_{\vec{\omega}}=$ $\prod_{i=1}^{m} \omega_{i}^{p / p_{i}}$. One says that $\vec{\omega}$ satisfies the $A_{\vec{p}}$ condition if

$$
\begin{aligned}
& \sup _{\mathrm{Q}}\left(\frac{1}{|Q|} \int_{Q} \prod_{i=1}^{m} \omega_{i}^{p / p_{i}}\right)^{1 / p} \prod_{i=1}^{m}\left(\frac{1}{|Q|} \int_{Q} \omega_{i}^{1-p_{i}^{\prime}}\right)^{1 / p_{i}^{\prime}} \\
& \quad<\infty
\end{aligned}
$$

when $p_{i}=1$ and $\left((1 /|Q|) \int_{Q} \omega_{i}^{1-p_{i}^{\prime}}\right)^{1 / p_{i}^{\prime}}$ is understood as $\left(\inf _{Q} \omega_{i}\right)^{-1}$.

Definition 2 (Dini $(a)$ condition). Suppose that $\omega(t)$ : $[0, \infty) \mapsto[0, \infty)$ is a nondecreasing function with $0<\omega(1)<$ $\infty$. For $a>0$, one says that $\omega \in \operatorname{Dini}(a)$, if

$$
|\omega|_{\operatorname{Dini}(a)}=\int_{0}^{1} \frac{\omega^{a}(t)}{t} d t<\infty .
$$

Definition 3 (kernels of type $\omega(t)$ ). For any $t \in(0, \infty)$, a locally integrable function $K_{t}\left(x, y_{1}, \ldots, y_{m}\right)$ defined away from the diagonal $x=y_{1}=\cdots=y_{m}$ in $\left(\mathbb{R}^{n}\right)^{m+1}$ is called a kernel of type $\omega(t)$, if there is a positive constant $A>0$, such that

$$
\begin{aligned}
& \left(\int_{0}^{\infty}\left|K_{t}\left(x, y_{1}, \ldots, y_{m}\right)\right|^{2} \frac{d t}{t}\right)^{1 / 2} \\
& \quad \leq \frac{A}{\left(\sum_{j=1}^{m}\left|x-y_{j}\right|\right)^{m n}} ; \\
& \left(\int_{0}^{\infty}\left|K_{t}\left(z, y_{1}, \ldots, y_{m}\right)-K_{t}\left(x, y_{1}, \ldots, y_{m}\right)\right|^{2} \frac{d t}{t}\right)^{1 / 2} \\
& \quad \leq \frac{A}{\left(\sum_{j=1}^{m}\left|x-y_{j}\right|\right)^{m n}} \omega\left(\frac{|z-x|}{\sum_{j=1}^{m}\left|x-y_{j}\right|}\right)
\end{aligned}
$$

whenever $|z-x| \leq(1 / 2) \max _{j=1}^{m}\left|x-y_{j}\right|$; and

$$
\begin{aligned}
& \left(\int_{0}^{\infty} \mid K_{t}\left(x, y_{1}, \ldots, y_{j}, \ldots, y_{m}\right)\right. \\
& \left.\quad-\left.K_{t}\left(x, y_{1}, \ldots, y_{j}^{\prime}, \ldots, y_{m}\right)\right|^{2} \frac{d t}{t}\right)^{1 / 2} \\
& \quad \leq \frac{A}{\left(\sum_{j=1}^{m}\left|x-y_{j}\right|\right)^{m n}} \omega\left(\frac{\left|y_{j}-y_{j}^{\prime}\right|}{\sum_{j=1}^{m}\left|x-y_{j}\right|}\right),
\end{aligned}
$$

whenever $\left|y_{j}-y_{j}^{\prime}\right| \leq(1 / 2) \max _{j=1}^{m}\left|x-y_{j}\right|$.

We say that $T$ is a multilinear square function with a kernel of type $\omega(t)$, if

$$
\begin{gathered}
T(\vec{f})(x)=\left(\int_{0}^{\infty} \mid \int_{\left(\mathbb{R}^{n}\right)^{m}} K_{t}\left(x, y_{1}, \ldots, y_{m}\right)\right. \\
\left.\quad \cdot \prod_{j=1}^{m} f_{j}\left(y_{j}\right) d y_{1}, \ldots,\left.d y_{m}\right|^{2} \frac{d t}{t}\right)^{1 / 2},
\end{gathered}
$$

whenever $x \notin \bigcap_{j=1}^{m}$ supp $f_{j}$ and each $f_{j} \in C_{c}^{\infty}\left(\mathbb{R}^{n}\right)$.
The corresponding multilinear maximal square function $T^{*}$ is defined by

$$
T^{*}(\vec{f})(x)=\sup _{\delta>0} T_{\delta}(\vec{f})(x),
$$

where

$$
\begin{gathered}
T_{\delta}(\vec{f})(x)=\left(\int_{0}^{\infty} \mid \int_{\sum_{i=1}^{m}\left|x-y_{i}\right|^{2}>\delta^{2}} K_{t}\left(x, y_{1}, \ldots, y_{m}\right)\right. \\
\left.\quad \cdot \prod_{j=1}^{m} f_{j}\left(y_{j}\right) d y_{1}, \ldots,\left.d y_{m}\right|^{2} \frac{d t}{t}\right)^{1 / 2} .
\end{gathered}
$$

We always assume that $T$ and $T^{*}$ can be extended to be a bounded operator from $L^{q_{1}} \times \cdots \times L^{q_{m}}$ to $L^{q}$ for some $1<$ $q, q_{1}, \ldots, q_{m}<\infty$ with $1 / q_{1}+\cdots+1 / q_{m}=1 / q$.

The aim of this paper is to study the bounded properties of multilinear square function $T$ and multilinear maximal square function $T^{*}$ with nonconvolution type kernels. It should be pointed out that the methods used in $[4,11,12]$ do not work for Littlewood-Paley operators with more general nonconvolution type kernels, for the reason that the estimates there rely heavily on the convolution type kernels and the well-known Marcinkiewicz function studied in [14].

We formulate the main results of this paper as follows.

Theorem 4. Let $T$ be a multilinear square function of type $\omega(t)$ and $\omega \in \operatorname{Dini}(1)$. Then, $T$ can be extended to be a bounded operator from $L^{1} \times \cdots \times L^{1}$ to $L^{1 / m, \infty}$.

Theorem 5. Let $T$ be a multilinear square function of type $\omega(t)$ and $\omega \in \operatorname{Dini}(1)$. Let $\vec{\omega} \in A_{\vec{p}}$ and $1 / p=1 / p_{1}+\cdots+1 / p_{m}$. Then, one has the following weighted estimates:

(i) If $1<p_{1}, \ldots, p_{m}<\infty$, then

$$
\|T \vec{f}\|_{L^{p}\left(v_{\vec{\omega}}\right)} \leq C \prod_{i=1}^{m}\left\|f_{i}\right\|_{L^{p_{i}}\left(\omega_{i}\right)} .
$$

(ii) If $1 \leq p_{1}, \ldots, p_{m}<\infty$, then

$$
\|T \vec{f}\|_{L^{p, \infty}\left(v_{\vec{\omega}}\right)} \leq C \prod_{i=1}^{m}\left\|f_{i}\right\|_{L^{p_{i}}\left(\omega_{i}\right)} .
$$

Theorem 6. Let $T^{*}$ be a multilinear maximal square function of type $\omega(t)$ and $\omega \in \operatorname{Dini}(1)$. Let $1 / p=1 / p_{1}+\cdots+1 / p_{m}$ and $\vec{\omega} \in A_{\vec{p}}$. Then, one has the following:

(i) If $1<p_{1}, \ldots, p_{m}<\infty$, then

$$
\left\|T^{*} \vec{f}\right\|_{L^{p}\left(v_{\vec{\omega}}\right)} \leq C \prod_{i=1}^{m}\left\|f_{i}\right\|_{L^{p_{i}}\left(\omega_{i}\right)} .
$$

(ii) If $1 \leq p_{1}, \ldots, p_{m}<\infty$, then

$$
\left\|T^{*} \vec{f}\right\|_{L^{p, \infty}\left(v_{\vec{\omega}}\right)} \leq C \prod_{i=1}^{m}\left\|f_{i}\right\|_{L^{p_{i}}\left(\omega_{i}\right)} .
$$

Remark 7. When $\omega(x)=x^{\gamma}$ for some $\gamma>0$, Theorems 4 and 5 were proved in [15]. 
Remark 8. The theory on multilinear Calderón-Zygmund operators has attracted much attention recently. In 2009, Maldonado and Naibo [16] established the weighted norm inequalities, with the Muckenhoupt weights, for bilinear Calderón-Zygmund operators of type $\omega(t)$. In 2014, Lu and Zhang [17] obtained some multiple-weighted norm inequalities for multilinear Calderón-Zygmund operators of type $\omega(t)$ and related operators.

The paper is organized as follows. Section 2 contains some preliminary information and proofs of Theorems 4-6. In Section 3, we establish the weighted strong and weak type boundedness of $T$ and $T^{*}$ on Morrey type spaces.

\section{Proofs of the Main Theorems}

First, we give the proof of Theorem 4.

Proof. The basic idea of the following arguments is essentially taken from $[15,17]$.

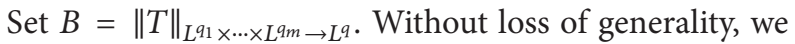
can assume that $\left\|f_{j}\right\|_{L^{1}}=1$, where $j=1, \ldots, m$. For any fixed number $\lambda>0$, we need to show that there is constant $C>0$ such that

$$
\begin{aligned}
\mid\{x & \left.\in \mathbb{R}^{n}:\left|T\left(f_{1}, \ldots, f_{m}\right)(x)\right|>\lambda\right\} \mid \\
& \leq C(A+B)^{1 / m} \lambda^{-1 / m} .
\end{aligned}
$$

We perform Calderón-Zygmund decomposition to each function $f_{j}$ at level $(\gamma \lambda)^{1 / m}$, where $\gamma$ is a positive number to be determined later. Then, we obtain a sequence of pairwise disjoint cubes $\left\{Q_{j, k_{j}}\right\}_{k_{j}=1}^{\infty}$ and decomposition $f_{j}=g_{j}+b_{j}=$ $g_{j}+\sum_{k_{j}} b_{j, k_{j}}$. Moreover, we have

(P1) $\operatorname{supp}\left(b_{j, k_{j}}\right) \subset Q_{j, k_{j}}$,

(P2) $\int_{\mathbb{R}^{n}} b_{j, k_{j}}(x) d x=0$,

(P3) $\int_{\mathbb{R}^{n}}\left|b_{j, k_{j}}(x)\right| d x \leq C(\gamma \lambda)^{1 / m}\left|Q_{j, k_{j}}\right|$,

(P4) $\sum_{k_{j}}\left|Q_{j, k_{j}}\right| \leq C(\gamma \lambda)^{1 / m}$,

(P5) $\left\|b_{j}\right\|_{L^{1}\left(\mathbb{R}^{n}\right)} \leq C$,

(P6) $\left\|g_{j}\right\|_{L^{s}\left(\mathbb{R}^{n}\right)} \leq C(\gamma \lambda)^{1 /\left(m s^{\prime}\right)}$ for $1 \leq s \leq \infty$.

Let $c_{j, k_{j}}$ be the center of cube $Q_{j, k_{j}}$ and let $l\left(Q_{j, k_{j}}\right)$ be its side length. For $j=1, \ldots, m$, set $\Omega^{*}=\bigcup_{j=1}^{m} \Omega_{j}^{*}$, where $\Omega_{j}^{*}=$ $\bigcup_{k_{j}} Q_{j, k_{j}}^{*}$ and $Q_{j, k_{j}}^{*}=8 \sqrt{n} Q_{j, k_{j}}$. And let

$$
\begin{gathered}
E_{1}=\left\{x \in \mathbb{R}^{n}:\left|T\left(g_{1}, g_{2}, \ldots, g_{m}\right)(x)\right|>\frac{\lambda}{2^{m}}\right\} ; \\
E_{2}=\left\{x \in \mathbb{R}^{n} \backslash \Omega^{*}:\left|T\left(b_{1}, g_{2}, \ldots, g_{m}\right)(x)\right|>\frac{\lambda}{2^{m}}\right\} ;
\end{gathered}
$$

$$
\begin{gathered}
E_{3}=\left\{x \in \mathbb{R}^{n} \backslash \Omega^{*}:\left|T\left(g_{1}, b_{2}, \ldots, g_{m}\right)(x)\right|>\frac{\lambda}{2^{m}}\right\} ; \\
\vdots \\
E_{2^{m}}=\left\{x \in \mathbb{R}^{n} \backslash \Omega^{*}:\left|T\left(b_{1}, b_{2}, \ldots, b_{m}\right)(x)\right|>\frac{\lambda}{2^{m}}\right\} .
\end{gathered}
$$

It follows from property (P4) that

$$
\left|\Omega^{*}\right| \leq \sum_{j=1}^{m}\left|\Omega_{j}^{*}\right| \leq C \sum_{j=1}^{m} \sum_{k_{j}}\left|Q_{j, k_{j}}\right| \leq C(\gamma \lambda)^{-1 / m}
$$

By the $L^{q_{1}} \times \cdots \times L^{q_{m}} \rightarrow L^{q, \infty}$ boundedness of $T$ and property (P6), we get

$$
\begin{aligned}
\left|E_{1}\right| & \leq\left(2^{m} B\right)^{q} \lambda^{-q}\left\|g_{1}\right\|_{L^{q_{1}\left(\mathbb{R}^{n}\right)}}^{q} \cdots\left\|g_{m}\right\|_{L^{q_{m}\left(\mathbb{R}^{n}\right)}}^{q} \\
& \leq C B^{q} \gamma^{q-1} \lambda^{-1 / m} .
\end{aligned}
$$

Thus, we have

$$
\begin{gathered}
\left|\left\{x \in \mathbb{R}^{n}:|T(\vec{f})(x)|>\lambda\right\}\right| \leq C \sum_{s=1}^{2^{m}}\left|E_{s}\right|+C\left|\Omega^{*}\right| \\
\leq C \sum_{s=2}^{2^{m}}\left|E_{s}\right|+C B^{q} \gamma^{q-1 / m} \lambda^{-1 / m}+C(\gamma \lambda)^{-1 / m} .
\end{gathered}
$$

To complete the proof, we need to estimate $\left|E_{s}\right|$ for $2 \leq s \leq$ $2^{m}$. Suppose that for some $1 \leq l \leq m$ there are $l$ bad functions and $m-l$ good functions appearing in $T\left(h_{1}, \ldots, h_{m}\right)$, where $h_{j} \in\left\{g_{j}, b_{j}\right\}$. For simplicity, we assume that the bad functions appear at the entries $1, \ldots, l$, and denote the corresponding term by $\left|E_{s}^{(l)}\right|$ to distinguish it from the other terms. That is, we will consider

$$
\begin{aligned}
\left|E_{s}^{(l)}\right| & =\mid\{x \\
& \in \mathbb{R}^{n} \backslash \Omega^{*}:\left|T\left(b_{1}, b_{2}, \ldots, b_{l}, g_{l+1}, \ldots, g_{m}\right)(x)\right| \\
> & \left.\frac{\lambda}{2^{m}}\right\} \mid
\end{aligned}
$$

and the other terms can be estimated similarly.

We will show that $\left|E_{s}^{(l)}\right| \leq C A(\gamma \lambda)^{-1 / m}$. Obviously, $T\left(b_{1}, b_{2}, \ldots, b_{l}, g_{l+1}, \ldots, g_{m}\right)(x)$ can be controlled by

$$
\sum_{k_{1}, \ldots, k_{l}}\left(\int_{0}^{\infty}\left|\int_{\mathbb{R}^{n m}} K_{t}\left(x, y_{1}, \ldots, y_{m}\right) \prod_{i=1}^{l} b_{i, k_{i}}\left(y_{i}\right) \prod_{i=l+1}^{m} g_{i}\left(y_{i}\right) d \vec{y}\right|^{2} \frac{d t}{t}\right)^{1 / 2}
$$


By properties (P4) and (P6) and Minkowski's inequality, we can further control the above term by

$$
\begin{aligned}
& \sum_{k_{1}, \ldots, k_{l}}\left(\int_{0}^{\infty}\left|\int_{\mathbb{R}^{n m}}\left(K_{t}\left(x, y_{1}, \ldots, y_{m}\right)-K_{t}\left(x, c_{1, k_{1}}, \ldots, y_{m}\right)\right) \prod_{i=1}^{l} b_{i, k_{i}}\left(y_{i}\right) \prod_{i=l+1}^{m} g_{i}\left(y_{i}\right) d \vec{y}\right|^{2} \frac{d t}{t}\right)^{1 / 2} \\
& \leq \sum_{k_{1}, \ldots, k_{l}} \int_{\mathbb{R}^{n m}}\left(\int_{0}^{\infty}\left|K_{t}\left(x, y_{1}, \ldots, y_{m}\right)-K_{t}\left(x, c_{1, k_{1}}, \ldots, y_{m}\right)\right|^{2} \frac{d t}{t}\right)^{1 / 2} \prod_{i=1}^{l} b_{i, k_{i}}\left(y_{i}\right) \prod_{i=l+1}^{m} g_{i}\left(y_{i}\right) d \vec{y} \\
& \leq C(\gamma \lambda)^{(m-l) / m} \sum_{k_{1}, \ldots, k_{l}} \int_{\mathbb{R}^{n m}}\left(\int_{0}^{\infty}\left|K_{t}\left(x, y_{1}, \ldots, y_{m}\right)-K_{t}\left(x, c_{1, k_{1}}, \ldots, y_{m}\right)\right|^{2} \frac{d t}{t}\right)^{1 / 2} \prod_{i=1}^{l} b_{i, k_{i}}\left(y_{i}\right) d \vec{y} .
\end{aligned}
$$

Let $Q_{r, k_{r}}^{i_{r}}=\left(2^{i_{r}+2} \sqrt{n} Q_{r, k_{r}}\right) \backslash\left(2^{i_{r}+1} \sqrt{n} Q_{r, k_{r}}\right)$ for $r=\bigcup_{i_{1}, \ldots, i_{l}=1}^{\infty} \bigcap_{r=1}^{l} Q_{r, k_{r}}^{i_{r}}$; see [17, p. 105]. Assume that $Q_{1, k_{1}}$ has the $1, \ldots, l$ and $i_{r}=1,2, \ldots$ It was proved that $\mathbb{R}^{n} \backslash Q^{*} \subset \quad$ smallest length in $\left\{Q_{i, k_{i}}\right\}_{i=1}^{l}$; then, by (9) one has

$$
\begin{aligned}
& \int_{\mathbb{R}^{n} \backslash Q^{*}}\left(\int_{0}^{\infty}\left|K_{t}\left(x, y_{1}, \ldots, y_{m}\right)-K_{t}\left(x, c_{1, k_{1}}, \ldots, y_{m}\right)\right|^{2} \frac{d t}{t}\right)^{1 / 2} d x \leq C \int_{\mathbb{R}^{n} \backslash Q^{*}} \frac{1}{\left(\sum_{j=1}^{m}\left|x-y_{j}\right|\right)^{m n}} \omega\left(\frac{\left|y_{1}-c_{1, k_{1}}\right|}{\sum_{j=1}^{m}\left|x-y_{j}\right|}\right) d x \\
& \quad \leq C \sum_{i_{1}, \ldots, i_{l}=1}^{\infty} \int_{\bigcap_{r=1}^{l} Q_{r, k_{r}}^{i_{r}}} \frac{1}{\left(\sum_{j=1}^{m}\left|x-y_{j}\right|\right)^{m n}} \omega\left(\frac{\left|y_{1}-c_{1, k_{1}}\right|}{\sum_{j=1}^{m}\left|x-y_{j}\right|}\right) d x \leq C \sum_{i_{1}, \ldots, i_{l}=1}^{\infty} \omega\left(2^{-i_{l}}\right) \int_{\bigcap_{r=1}^{l} Q_{r, k_{r}}^{i_{r}}} \frac{1}{\left(\sum_{j=1}^{m}\left|x-y_{j}\right|\right)^{m n}} d x .
\end{aligned}
$$

This together with Chebyshev's inequality gives

$$
\begin{aligned}
\left|E_{s}^{(l)}\right| & \leq \frac{2^{m}}{\lambda} \int_{\mathbb{R}^{n} \backslash Q^{*}}\left|T\left(b_{1}, b_{2}, \ldots, b_{l}, g_{l+1}, \ldots, g_{m}\right)(x)\right| d x \\
& \leq \frac{2^{m}}{\lambda}(\gamma \lambda)^{(m-l) / m} \int_{\mathbb{R}^{n} \backslash Q^{*}} \sum_{k_{1}, \ldots, k_{l}} \int_{\mathbb{R}^{n m}}\left(\int_{0}^{\infty}\left|K_{t}\left(x, y_{1}, \ldots, y_{m}\right)-K_{t}\left(x, c_{1, k_{1}}, \ldots, y_{m}\right)\right|^{2} \frac{d t}{t}\right)^{1 / 2} \prod_{i=1}^{l} b_{i, k_{i}}\left(y_{i}\right) d \vec{y} d x \\
& \leq \frac{2^{m}}{\lambda}(\gamma \lambda)^{(m-l) / m} \sum_{k_{1}, \ldots, k_{l} i_{1}, \ldots, i_{l}=1} \omega\left(2^{-i_{l}}\right) \int_{\left(\mathbb{R}^{n}\right)^{m-l}}^{\infty} \int_{\prod_{r=1}^{l} Q_{r, k_{r}}} \int_{\prod_{r=1}^{l} Q_{r, k_{r}}^{i_{r}}} \frac{d x}{\left(\sum_{j=1}^{m}\left|x-y_{j}\right|\right)^{m n}} \prod_{i=1}^{l} b_{i, k_{i}}\left(y_{i}\right) d \vec{y},
\end{aligned}
$$

where $\prod_{r=1}^{l} Q_{r, k_{r}}=Q_{1, k_{1}} \times \cdots \times Q_{l, k_{l}}$.

Now, by repeating the same arguments as in [17, p. 106108], we can easily obtain $\left|E_{s}^{(l)}\right| \leq C A \gamma(\gamma \lambda)^{-1 / m}$. Thus, setting $\gamma=(A+B)^{-1}$, we get

$$
\begin{aligned}
\mid\{x & \left.\in \mathbb{R}^{n}:|T(\vec{f})(x)|>\lambda\right\} \mid \\
& \leq C A \gamma(\gamma \lambda)^{-1 / m}+C B^{q} \gamma^{q-1 / m} \lambda^{-1 / m}+C(\gamma \lambda)^{-1 / m} \\
& =C(A+B)^{1 / m} \lambda^{-1 / m} .
\end{aligned}
$$

This completes the proof of Theorem 4.

In order to prove Theorem 5, the following lemmas are needed.
Lemma 9 (see [15]). Suppose that supp $f_{i} \subset B(0, R)$; then, there is a constant $C<\infty$ such that, for all $|x|>2 R$, the following inequality holds:

$$
T(\vec{f})(x) \leq C \mathscr{M}(\vec{f})(x)
$$

Lemma 10. Let $T$ be a multilinear square function of type $\omega(t)$ and $\omega \in \operatorname{Dini}(1)$. For any $0<\delta<1 / m$, there is a constant $C<\infty$ such that for any bounded and compact supported $f_{j}$, $j=1, \ldots, m$, the following inequality holds:

$$
M_{\delta}^{\sharp} T(\vec{f})(x) \leq C \mathscr{M}(\vec{f})(x) .
$$


Proof. The proof of Lemma 10 involves a routine application of the method used in Lemma 4.1 in [15]. For the sake of similarity, we sketch the proof. Given $0<\delta<1 / m$, for a fixed point $x \in \mathbb{R}^{n}$ and a cube $Q \ni x$, it is sufficient to show that there exists a constant $c_{Q}$ such that

$$
\left(\frac{1}{|Q|} \int_{Q}\left|T(\vec{f})(z)-c_{Q}\right|^{\delta} d z\right)^{1 / \delta} \leq C \mathscr{M} \vec{f}(x)
$$

For each $i=1, \ldots, m$, let $\vec{\alpha}=\left(\alpha_{1}, \ldots, \alpha_{m}\right)$ satisfying $\alpha_{i}=$ 0 or $\infty$. We next introduce two notations:

$$
\begin{aligned}
c_{\mathrm{Q}, t} & =\sum_{\vec{\alpha}, \vec{\alpha} \neq \overrightarrow{0}} \int_{\mathbb{R}^{n m}} K_{t}\left(x, y_{1}, \ldots, y_{m}\right) \prod_{j=1}^{m} f_{j}\left(y_{j}\right) d \vec{y}, \\
c_{\mathrm{Q}} & =\left(\int_{0}^{\infty}\left|c_{\mathrm{Q}, t}\right|^{2} \frac{d t}{t}\right)^{1 / 2} .
\end{aligned}
$$

Therefore,

$$
\left(\frac{1}{|Q|} \int_{Q}\left|T(\vec{f})(z)-c_{Q}\right|^{\delta} d z\right)^{1 / \delta} \leq C I_{\overrightarrow{0}}+C \sum_{\vec{\alpha} \neq 0} I_{\vec{\alpha}},
$$

where

$$
\begin{aligned}
& I_{\overrightarrow{0}}=\left(\frac{1}{|Q|} \int_{Q}\left(\int_{0}^{\infty}\left|\int_{\mathbb{R}^{n m}} K_{t}(z, \vec{y}) \prod_{j=1}^{m} f_{j}^{0}\left(y_{j}\right) d \vec{y}\right|^{2} \frac{d t}{t}\right)^{\delta / 2} d z\right)^{1 / \delta}, \\
& I_{\vec{\alpha}}=\sum_{\vec{\alpha}, \vec{\alpha} \neq \overrightarrow{0}}\left(\frac{1}{|Q|} \int_{Q}\left(\int_{0}^{\infty}\left|\int_{\mathbb{R}^{n m}}\left(K_{t}(z, \vec{y})-K_{t}(x, \vec{y})\right) \prod_{j=1}^{m} f_{j}^{\alpha_{j}}\left(y_{j}\right) d \vec{y}\right|^{2} \frac{d t}{t}\right)^{\delta / 2} d z\right)^{1 / \delta} .
\end{aligned}
$$

By using the boundedness of $T$, we get immediately that $I_{\overrightarrow{0}} \leq C \mathscr{M}(\vec{f})(x)$.

Using the smooth condition (8), we obtain

$$
\begin{aligned}
& \int_{\mathbb{R}^{n m}}\left(\int_{0}^{\infty}\left|K_{t}(z, \vec{y})-K_{t}(x, \vec{y})\right|^{2} \frac{d t}{t}\right)^{1 / 2} \\
& \cdot \prod_{j=1}^{m}\left|f_{j}^{\alpha_{j}}\left(y_{j}\right)\right| d \vec{y} \leq C \int_{\mathrm{Q}(x, \delta)^{l}} \prod_{i \in \mathscr{F}}\left|f_{i}^{0}\left(y_{i}\right) d y_{i}\right| \\
& \cdot \sum_{k=1}^{\infty} \int_{\left(Q_{k}\right)^{m-l}} \frac{A}{\left(\sum_{i \in \mathscr{F}^{c}}\left|z-y_{i}\right|\right)^{m n}} \omega\left(\frac{|z-x|}{\sum_{i \in \mathscr{F}^{c}}\left|z-y_{i}\right|}\right)
\end{aligned}
$$

$$
\begin{aligned}
& \cdot \prod_{i \in \mathscr{F}^{c}}\left|f_{i}^{\infty}\left(y_{i}\right)\right| d y_{i} \leq C \int_{Q(x, \delta)^{l}} \prod_{i \in \mathscr{I}}\left|f_{i}^{0}\left(y_{i}\right) d y_{i}\right| \\
& \cdot \sum_{k=1}^{\infty} \int_{\left(Q_{k}\right)^{m-l}} \frac{\omega\left(2^{-k}\right)}{\left|2^{k} Q\right|^{m}} \prod_{i \in \mathscr{F}^{c}}\left|f_{i}^{\infty}\left(y_{i}\right)\right| d y_{i} \\
& \leq C \sum_{k=1}^{\infty} \omega\left(2^{-k}\right) \frac{1}{\left|2^{k} Q\right|^{m}} \prod_{i \in \mathscr{I}} \int_{Q(x, \delta)}\left|f_{i}^{0}\left(y_{i}\right)\right| d y_{i} \\
& \cdot \prod_{i \in \mathscr{F}^{c}} \int_{2^{k} Q}\left|f_{i}^{\infty}\left(y_{i}\right)\right| d y_{i} \leq C|\omega|_{\operatorname{Dini}(1)} \mathscr{M}(\vec{f})(x) .
\end{aligned}
$$

Then, by Minkowski's inequality, it yields that

$$
I_{\vec{\alpha}} \leq C\left(\frac{1}{|Q|} \int_{Q}\left(\int_{\mathbb{R}^{n m}}\left(\int_{0}^{\infty}\left|K_{t}(z, \vec{y})-K_{t}(x, \vec{y})\right|^{2} \frac{d t}{t}\right)^{1 / 2} \prod_{j=1}^{m}\left|f_{j}^{\alpha_{j}}\left(y_{j}\right)\right| d \vec{y}\right)^{\delta} d z\right)^{1 / \delta} \leq C|\omega|_{\operatorname{Dini}(1)} \mathscr{M}(\vec{f})(x) .
$$

Thus, we have finished the proof of Lemma 10.

Proof of Theorem 5. Theorem 5 follows from using Lemmas 9 and 10 and repeating the same steps as in [15], here, we omit the proof.

To prove Theorem 6, we need some preliminary lemmas.

Lemma 11 (see [18]). If $\omega \in A_{p}$ and $p \geq 1$, then $M$ maps from $L^{p, \infty}(\omega)$ to $L^{p, \infty}(\omega)$.
Lemma 12 (see $[13])$. Let $\vec{p}=\left(p_{1}, \ldots, p_{m}\right)$ with $1 / p=1 / p_{1}+$ $\cdots+1 / p_{m}$ and $1 \leq p_{1}, \ldots, p_{m}$.

(1) If $1<p_{j}<\infty$ for all $j=1, \ldots, m$, then $\mathscr{M}$ is bounded from $L^{p_{1}}\left(\omega_{1}\right) \times \cdots \times L^{p_{m}}\left(\omega_{m}\right)$ to $L^{p}\left(\nu_{\vec{\omega}}\right)$ if and only if $\vec{\omega}=\left(\omega_{1}, \ldots, \omega_{m}\right) \in A_{\vec{p}}$.

(2) If $1 \leq p_{j}<\infty$ for all $j=1, \ldots, m$, then $\mathscr{M}$ is bounded from $L^{p_{1}}\left(\omega_{1}\right) \times \cdots \times L^{p_{m}}\left(\omega_{m}\right)$ to $L^{p, \infty}\left(\nu_{\vec{\omega}}\right)$ if $\vec{\omega}=\left(\omega_{1}, \ldots, \omega_{m}\right) \in A_{\vec{p}}$. 
Lemma 13 (see [19]). If $\omega \in A_{p}$ and $p>1$, then $M$ mapsfrom $L^{p}(\omega)$ to $L^{p}(\omega)$.

Lemma 14. Let $T$ be a multilinear square function of type $\omega(t)$ and $\omega \in \operatorname{Dini}(1)$. For any $\eta>0$, there is a constant $C<\infty$ depending on $\eta$ such that for all $\vec{f}$ in any product of $L^{q_{j}}\left(\mathbb{R}^{n}\right)$ spaces, with $1 \leq q_{j}<\infty$, the following inequality holds for all $x \in \mathbb{R}^{n}$ :

$$
T^{*}(\vec{f})(x) \leq C\left(M_{\eta}(T(\vec{f}))(x)+\mathscr{M}(\vec{f})(x)\right) .
$$

Proof. The basic idea is due to $[18,20]$. Set $U_{\delta}=\{\vec{y} \in$ $\left.(Q(x, \delta))^{m}: \sum_{i=1}^{m}\left|x-y_{i}\right|^{2}>\delta^{2}\right\}$. For a fixed point $x$ and a cube $Q(x, \delta)$ centered at $x$ with radius $\delta$, it is clear that

$$
\begin{aligned}
& \left|T^{*}(\vec{f})(x)\right| \\
& \quad \leq \sup _{\delta>0}\left(\int_{0}^{\infty}\left|\int_{U_{\delta}} K_{t}(x, \vec{y}) \prod_{i=1}^{m} f_{i}\left(y_{i}\right) d y_{i}\right|^{2} \frac{d t}{t}\right)^{1 / 2} \\
& \quad+\sup _{\delta>0}\left(\int_{0}^{\infty}\left|\int_{\left((Q(x, \delta))^{m}\right)^{c}} K_{t}(x, \vec{y}) \prod_{i=1}^{m} f_{i}\left(y_{i}\right) d y_{i}\right|^{2} \frac{d t}{t}\right)^{1 / 2} .
\end{aligned}
$$

By using the size condition (7) and Minkowski's inequality, we get

$$
\begin{aligned}
& \left(\int_{0}^{\infty}\left|\int_{U_{\delta}} K_{t}(x, \vec{y}) \prod_{i=1}^{m} f_{i}\left(y_{i}\right) d y_{i}\right|^{2} \frac{d t}{t}\right)^{1 / 2} \\
& \quad \leq C\left|\int_{U_{\delta}}\left(\int_{0}^{\infty}\left|K_{t}(z, \vec{y})\right|^{2} \frac{d t}{t}\right)^{2} \prod_{i=1}^{m} f_{i}\left(y_{i}\right) d y_{i}\right| \\
& \quad \leq C\left|\int_{U_{\delta}} \frac{A}{\left(\sum_{i=1}^{m}\left|y_{i}-x\right|\right)^{m n}} \prod_{i=1}^{m} f_{i}\left(y_{i}\right) d y_{i}\right| \\
& \leq C \prod_{i=1}^{m} \frac{1}{\delta^{n}} \int_{\mathrm{Q}(x, \delta)} f_{i}\left(y_{i}\right) d y_{i} \leq C \mathscr{M}(\vec{f})(x) .
\end{aligned}
$$

We are ready to estimate the second term. Set $\vec{f}^{0}=$ $\left(f_{1} \chi_{\mathrm{Q}}, \ldots, f_{m} \chi_{\mathrm{Q}}\right)$. For any $z \in Q(x, \delta / 2)$, we introduce an operator $\widetilde{T}_{\delta}$ :

$$
\begin{aligned}
& \widetilde{T}_{\delta}(\vec{f})(z) \\
& \quad=\left(\int_{0}^{\infty}\left|\int_{\left((\mathrm{Q}(x, \delta))^{m}\right)^{c}} K_{t}(z, \vec{y}) \prod_{i=1}^{m} f_{i}\left(y_{i}\right) d y_{i}\right|^{2} \frac{d t}{t}\right)^{1 / 2} \\
& \quad \leq T(\vec{f})(z)+T\left(\vec{f}^{0}\right)(z) .
\end{aligned}
$$

Let $R_{\mathscr{I}}$ be the sets in $\left(\mathbb{R}^{n}\right)^{m}$, where $\mathscr{I}:=\left\{i_{1}, \ldots, i_{l}\right\} \subseteq$ $\{1, \ldots, m\}$, such that for $\vec{y}=\left(y_{1}, \ldots, y_{m}\right) \in R_{\mathscr{I}}$ we have $i \in \mathscr{I}$ if and only if $\left|x-y_{i}\right| \leq \delta$. Using the smooth condition (8), we obtain that

$$
\begin{aligned}
& \left|\widetilde{T}_{\delta}(\vec{f})(x)-\widetilde{T}_{\delta}(\vec{f})(z)\right| \\
& \leq C\left(\int _ { 0 } ^ { \infty } \left(\int_{\left((Q(x, \delta))^{m}\right)^{c}}\left|K_{t}(z, \vec{y})-K_{t}(x, \vec{y})\right|\right.\right. \\
& \left.\left.\cdot \prod_{i=1}^{m}\left|f_{i}\left(y_{i}\right)\right| d y_{i}\right)^{2} \frac{d t}{t}\right)^{1 / 2} \\
& \leq C \int_{\left((Q(x, \delta))^{m}\right)^{c}}\left(\int_{0}^{\infty}\left|K_{t}(z, \vec{y})-K_{t}(x, \vec{y})\right|^{2} \frac{d t}{t}\right)^{1 / 2} \\
& \cdot \prod_{i=1}^{m}\left|f_{i}\left(y_{i}\right)\right| d y_{i} \leq C \sum_{\vec{y} \in R_{\mathcal{F}}} \int_{\mathrm{Q}(x, \delta)^{\prime}} \prod_{i \in \mathcal{F}}\left|f_{i}^{0}\left(y_{i}\right)\right| d y_{i} \\
& \cdot \sum_{k=1}^{\infty} \int_{\left(Q_{k}\right)^{m-l}} \frac{A}{\left(\sum_{i \in \mathscr{F}^{c}}\left|z-y_{i}\right|\right)^{m n}} \omega\left(\frac{|z-x|}{\sum_{i \in \mathscr{F}^{c}}\left|z-y_{i}\right|}\right) \\
& \cdot \prod_{i \in \mathscr{I}^{c}}\left|f_{i}^{\infty}\left(y_{i}\right)\right| d y_{i} \leq C \sum_{\vec{y} \in R_{\mathcal{F}}} \int_{\mathrm{Q}(x, \delta)^{l}} \prod_{i \in \mathscr{I}}\left|f_{i}^{0}\left(y_{i}\right)\right| d y_{i} \\
& \cdot \sum_{k=1}^{\infty} \int_{\left(Q_{k}\right)^{m-l}} \frac{\omega\left(2^{-k}\right)}{\left|2^{k} Q\right|^{m}} \prod_{i \in \mathscr{I}^{c}}\left|f_{i}^{\infty}\left(y_{i}\right)\right| d y_{i} \\
& \leq C \sum_{\vec{y} \in R_{\mathscr{F}}} \sum_{k=1}^{\infty} \omega\left(2^{-k}\right) \frac{1}{\left|2^{k} Q\right|^{m}} \prod_{i \in \mathscr{Y}} \int_{Q(x, \delta)}\left|f_{i}^{0}\left(y_{i}\right)\right| d y_{i} \\
& \cdot \prod_{i \in \mathscr{I}^{c}} \int_{2^{k} Q}\left|f_{i}^{\infty}\left(y_{i}\right)\right| d y_{i} \leq C|\omega|_{\operatorname{Dini}(1)} \mathscr{M}(\vec{f})(x),
\end{aligned}
$$

where $\mathbb{Q}_{k}=\left(2^{k} Q\right) \backslash\left(2^{k-1} Q\right)$, for $k=1,2, \ldots, \infty$.

Thus, we obtain

$$
\begin{aligned}
& \left(\int_{0}^{\infty}\left|\int_{\left((Q(x, \delta))^{m}\right)^{c}} K(x, \vec{y}) \prod_{i=1}^{m} f_{i}\left(y_{i}\right) d y_{i}\right|^{2} \frac{d t}{t}\right)^{1 / 2} \\
& \quad \leq C \mathscr{M}(\vec{f})(x)+|T(\vec{f})(z)|+\left|T\left(\vec{f}^{0}\right)(z)\right| .
\end{aligned}
$$

Raising the above inequality to the power $\eta$, integrating over $z \in Q(x, \delta / 2)$, and dividing by $|Q|$, we conclude that

$$
\begin{aligned}
& \left(\int_{0}^{\infty}\left|\int_{\left((\mathrm{Q}(x, \delta))^{m}\right)^{c}} K(x, \vec{y}) \prod_{i=1}^{m} f_{i}\left(y_{i}\right) d y_{i}\right|^{2} \frac{d t}{t}\right)^{\eta / 2} \\
& \leq C(\mathscr{M}(\vec{f})(x))^{\eta}+M\left(|T(\vec{f})|^{\eta}\right)(x) \\
& \quad+\frac{1}{|Q|} \int_{Q}\left|T\left(\vec{f}^{0}\right)(z)\right|^{\eta} d z .
\end{aligned}
$$


Next we estimate the last term in (42). By Theorem 4, we know that $T$ is bounded from $L^{1} \times \cdots \times L^{1}$ to $L^{1 / m, \infty}$; then we can deduce that

$$
\begin{aligned}
& \int_{Q}\left|T\left(\vec{f}^{0}\right)(z)\right|^{\eta} d z=m \eta \int_{0}^{\infty} \lambda^{m \eta-1} \mid\{z \\
& \left.\in Q:\left|T\left(\vec{f}^{0}\right)(z)\right|^{1 / m}>\lambda\right\} \mid d \lambda \\
& \leq m \eta \int_{0}^{\infty} \lambda^{m \eta-1} \\
& \cdot \min \left\{|Q|, \frac{c}{\lambda}\left(\prod_{j=1}^{m}\left\|f_{j} \chi_{Q(x, \delta)}\right\|_{L^{1}}\right)^{1 / m}\right\} d \lambda .
\end{aligned}
$$

Letting $R=c\left(\prod_{j=1}^{m}\left\|f_{j} \chi_{\mathrm{Q}(x, \delta)}\right\|_{L^{1}}\right)^{1 / m}$, we obtain that

$$
\begin{aligned}
& \frac{1}{|Q|} \int_{Q}\left|T\left(\vec{f}^{0}\right)(z)\right|^{\eta} d z \\
& \quad \leq \frac{C}{|Q|}\left(\int_{0}^{R /|Q|} \lambda^{m \eta-1}|Q| d \lambda+\int_{R /|Q|}^{\infty} \lambda^{m \eta-1} R d \lambda\right) \\
& \quad \leq C R^{m \eta}|Q|^{1-m \eta} \leq C\left(\prod_{j=1}^{m} \frac{1}{|Q|} \int_{Q}\left|f_{j}\left(y_{j}\right)\right| d y_{j}\right)^{\eta} \\
& \quad \leq C(\mathscr{M}(\vec{f})(x))^{\eta},
\end{aligned}
$$

where we have used the fact $m \eta<1$ (it suffices to prove the lemma for $\eta$ arbitrarily small). Finally, if we insert estimate (44) into (42) and raise to the power $1 / \eta$, we obtain the desired estimate. This finishes the proof of Lemma 14.

Proof of Theorem 6. Theorem 6 follows by using Lemmas 13 and 14 . Using the pointwise estimate for $T^{*}$ in Lemma 14, we obtain that

$$
\begin{aligned}
& \left\|T^{*}(\vec{f})\right\|_{L^{p}\left(v_{\vec{\omega}}\right)} \\
& \quad \leq C\left(\left\|M_{\eta}(T(\vec{f}))\right\|_{L^{p}\left(v_{\vec{\omega}}\right)}+\|\mathscr{M}(\vec{f})\|_{L^{p}\left(v_{\vec{\omega}}\right)}\right) .
\end{aligned}
$$

Notice that $\nu_{\vec{\omega}} \in A_{m p}$ for all $\vec{\omega} \in A_{\vec{p}}$ (see [13, Theorem 3.6]). By Lemma 13, we have

$$
\left\|M_{\eta}(T(\vec{f}))\right\|_{L^{p}\left(v_{\vec{\omega}}\right)} \leq C\|T(\vec{f})\|_{L^{p}\left(v_{\vec{\omega}}\right)} .
$$

Thus, we obtain the desired estimates by applying Theorem 5 and Lemma 12:

$$
\left\|T^{*}(\vec{f})\right\|_{L^{p}\left(v_{\vec{\omega}}\right)} \leq C \prod_{j=1}^{m}\left\|f_{j}\right\|_{L^{p_{j}}\left(\omega_{j}\right)} .
$$

If we use Lemma 11, by using the same arguments, we can get the weak type estimates; we omit its proof here.

\section{Weighted Boundedness on Morrey Type Spaces}

The classical Morrey space was first introduced by Morrey in [21] to study the local behavior of solutions to second order elliptic partial differential equations. Later, Komori and Shirai [22] introduced the weighted Morrey space $\mathscr{L}^{p, k}(\omega)$ for $1 \leq p<\infty$ and investigated the boundedness of classical operators, including Hardy-Littlewood maximal operator, Calderón-Zygmund operator, and fractional integral operator. In order to deal with the multilinear case $m \geq 2$, Wang and $\mathrm{Yi}$ [23] extended the range $1 \leq p<\infty$ to $0<p<\infty$.

Motivated by the works on multilinear CalderónZygmund operators and multilinear square functions, as demonstrated in $[4,12,15,24-26]$, we are going to study the boundedness of multilinear square function of type $\omega(t)$ on weighted Morrey type spaces.

Let $0<p<\infty$ and $0<k<1$ and let $\omega$ be a weighted function on $\mathbb{R}^{n}$. Then, the weighted Morrey space $\mathscr{L}^{p, k}$ is defined by

$$
\mathscr{L}^{p, k}=\left\{f \in L_{\mathrm{loc}}^{p}(\mu):\|f\|_{\mathscr{L}^{p, k}}<\infty\right\},
$$

where

$$
\|f\|_{\mathscr{L}^{p, k}}=\sup _{Q}\left(\frac{1}{\omega(Q)^{k}} \int_{Q}|f(x)|^{p} \omega(x) d x\right)^{1 / p} .
$$

Furthermore, the weighted weak Morrey space $W \mathscr{L}^{p, k}$ is defined by

$$
W \mathscr{L}^{p, k}=\left\{f \text { measurable }:\|f\|_{W \mathscr{L}^{p, k}}<\infty\right\},
$$

where

$$
\begin{aligned}
& \|f\|_{W \mathscr{L}^{p, k}} \\
& \quad=\sup _{\mathrm{Q}} \sup _{\lambda>0} \frac{1}{\omega(Q)^{k / p}} \lambda \cdot(\{x \in Q:|f(x)|>\lambda\})^{1 / p} .
\end{aligned}
$$

The main results in this section are the following Theorem.

Theorem 15. Let $T$ be a multilinear square function of type $\omega(t)$ and $\omega \in \operatorname{Dini}(1)$. Suppose that $\vec{\omega} \in A_{\vec{p}}$ and $\nu_{\vec{\omega}}=$ $\prod_{i=1}^{m} \omega_{i}^{p / p_{i}}$ with $1 / p=1 / p_{1}+\cdots+1 / p_{m}$. For $1 / m<p<\infty$ and $0<k<1$, the following two weighted inequalities hold:

(i) If $1<p_{1}, \ldots, p_{m}<\infty$, then

$$
\left\|T^{*}(\vec{f})\right\|_{\mathscr{L}^{p, k}\left(v_{\vec{\omega}}\right)} \leq C \prod_{j=1}^{m}\left\|f_{j}\right\|_{\mathscr{L}^{p j, k}\left(w_{j}\right)} .
$$

(ii) If $1 \leq p_{1}, \ldots, p_{m}<\infty$ and $\min \left\{p_{1}, \ldots, p_{m}\right\}=1$, then

$$
\left\|T^{*}(\vec{f})\right\|_{W \mathscr{L}^{p, k}\left(v_{\vec{\omega}}\right)} \leq C \prod_{j=1}^{m}\left\|f_{j}\right\|_{\mathscr{L}^{p_{j}, k}\left(w_{j}\right)} .
$$

Remark 16. Theorem 15 also holds with $T^{*}$ replaced by $T$. 
In order to prove Theorem 15, we will use the following lemmas.

Lemma 17 (see [23]). Let $m \geq 2, p_{1}, \ldots, p_{m} \in[1, \infty)$, and $p \in(0, \infty)$ with $1 / p=1 / p_{1}+\cdots+1 / p_{m}$. Assume that $\omega_{1}, \ldots, \omega_{m} \in A_{\infty}$ and set $\nu_{\vec{\omega}}=\prod_{i=1}^{m} \omega_{i}^{p / p_{i}}$. Then, for any ball $B$, there exists a constant $C>0$ such that

$$
\prod_{i=1}^{m}\left(\int_{B} \omega_{i}(x) d x\right)^{p / p_{i}} \leq C \int_{B} \nu_{\vec{\omega}}(x) d x .
$$

Lemma 18 (see [27]). Let $\omega \in A_{p}$ with $1 \leq p<\infty$. Then, for any ball $B$, there exists an absolute constant $C>0$ such that

$$
\omega(2 B) \leq C \omega(B) .
$$

Lemma 19 (see [27]). Let $\omega \in A_{\infty}$. Then, for any ball $B$ and all measurable subsets $E$ of $B$, there exists $\delta>0$ such that

$$
\frac{\omega(E)}{\omega(B)} \leq C\left(\frac{|E|}{|B|}\right)^{\delta}
$$

Now we are in the position to prove Theorem 15.

Proof. First, let us prove (i). For a fixed point $x_{0} \in \mathbb{R}^{n}$ and a ball $B \ni x_{0}$, we split each $f_{i}$ as $f_{i}=f_{i}^{0}+f_{i}^{\infty}$, where $f_{i}^{0}=f_{i} \chi_{2 B}$ for $i=1, \ldots, m$. Write

$$
\prod_{i=1}^{m} f_{i}\left(y_{i}\right)=\prod_{i=1}^{m} f_{i}^{0}\left(y_{i}\right)+\sum_{\vec{\alpha}, \vec{\alpha} \neq \overrightarrow{0}} f_{1}^{\alpha_{1}}\left(y_{1}\right) \cdots f_{m}^{\alpha_{m}}\left(y_{m}\right),
$$

where $\vec{\alpha}=\left(\alpha_{1}, \ldots, \alpha_{m}\right), \alpha_{i}=0$ or $\infty$ for $i=1, \ldots, m$.
Then, we have

$$
\begin{aligned}
& \left(\frac{1}{v_{\vec{\omega}}(B)^{k / p}} \int_{B}\left|T^{*}\left(f_{1}, \ldots, f_{m}\right)(x)\right|^{p} \nu_{\vec{\omega}}(x) d x\right)^{1 / p} \\
& \leq C \frac{1}{\nu_{\vec{\omega}}(B)^{k / p}}\left(\int_{B}\left|T^{*}\left(f_{1}^{0}, \ldots, f_{m}^{0}\right)(x)\right|^{p}\right. \\
& \left.\cdot \nu_{\vec{\omega}}(x) d x\right)^{1 / p} \\
& +\sum_{\vec{\alpha}, \vec{\alpha} \neq \overrightarrow{0}} \frac{1}{\nu_{\vec{\omega}}(B)^{k / p}}\left(\int_{B}\left|T^{*}\left(f_{1}^{\alpha_{1}}, \ldots, f_{m}^{\alpha_{m}}\right)(x)\right|^{p}\right. \\
& \left.\cdot \nu_{\vec{\omega}}(x) d x\right)^{1 / p}=C\left(I_{\overrightarrow{0}}+\sum_{\vec{\alpha}, \vec{\alpha} \neq \overrightarrow{0}} I_{\vec{\alpha}}\right) .
\end{aligned}
$$

It was shown in $\left[13\right.$, Theorem 3.6] that $\nu_{\vec{\omega}} \in A_{m p}$. This fact together with Theorem 6(i) and Lemmas 18 and 17 yields that

$$
\begin{aligned}
I_{\overrightarrow{0}} & <\frac{1}{\nu_{\vec{\omega}}(B)^{k / p}} \prod_{i=1}^{m}\left(\int_{B}\left|f_{i}(x)\right|^{p_{i}} \omega_{i}(x) d x\right)^{1 / p_{i}} \\
& \leq C \prod_{i=1}^{m}\left\|f_{i}\right\|_{\mathscr{L}^{p_{i}, k}\left(w_{i}\right)} \frac{\prod_{i=1}^{m} \omega_{i}(2 B)^{k / p_{i}}}{\nu_{\vec{\omega}}(B)^{k / p}} \\
& \leq C \prod_{i=1}^{m}\left\|f_{i}\right\|_{\mathscr{L}^{p_{i}, k}\left(w_{i}\right)} \frac{\nu_{\vec{\omega}}(2 B)^{k / p}}{\nu_{\vec{\omega}}(B)^{k / p}} \leq C \prod_{i=1}^{m}\left\|f_{i}\right\|_{\mathscr{L}^{p_{i}, k}\left(w_{i}\right)} .
\end{aligned}
$$

We now estimate $I_{\vec{\alpha}}$ with $\vec{\alpha}=(\infty, \ldots, \infty)$. For any $x \in B$, by Minkowski's inequality, the size condition (7), and Lemma 17, we have

$$
\begin{aligned}
& \left|T^{*}\left(f_{1}^{\infty}, \ldots, f_{m}^{\infty}\right)(x)\right|=\sup _{\delta>0}\left(\int_{0}^{\infty}\left|\int_{\sum_{i=1}^{m}\left|x-y_{i}\right|^{2}>\delta^{2}} K_{t, \delta}\left(x, y_{1}, \ldots, y_{m}\right) \prod_{i=1}^{m} f_{i}^{\infty}\left(y_{i}\right) d \vec{y}\right|^{2} \frac{d t}{t}\right)^{1 / 2} \\
& \leq C \int_{\mathbb{R}^{n m}}\left(\int_{0}^{\infty}\left|K_{t}(x, \vec{y})\right|^{2} \frac{d t}{t}\right)^{1 / 2} \prod_{i=1}^{m} f_{i}^{\infty}\left(y_{i}\right) d \vec{y} \\
& \leq C \sum_{j=1}^{\infty} \int_{\left(2^{j+1} B \backslash 2^{j} B\right)^{m}} \frac{1}{\left(\sum_{i=1}^{m}\left|x-y_{i}\right|\right)^{m n}} \prod_{i=1}^{m} f_{i}\left(y_{i}\right) d \vec{y} \leq C \sum_{j=1}^{\infty} \prod_{i=1}^{m} \frac{1}{\left|2^{j+1} B\right|} \int_{2^{j+1} B}\left|f_{i}\left(y_{i}\right)\right| d y_{i} \\
& \leq C \sum_{j=1}^{\infty} \prod_{i=1}^{m} \frac{1}{\left|2^{j+1} B\right|}\left(\int_{2^{j+1} B}\left|f_{i}\left(y_{i}\right)\right|^{p_{i}} \omega_{i}\left(y_{i}\right) d y_{i}\right)^{1 / p_{i}}\left(\int_{2^{j+1} B} \omega_{i}\left(y_{i}\right)^{1-p_{i}^{\prime}} d y_{i}\right)^{1 / p_{i}^{\prime}} \\
& \leq C \sum_{j=1}^{\infty} \frac{1}{\left|2^{j+1} B\right|^{m}} \frac{\left|2^{j+1} B\right|^{1 / p+\sum_{i=1}^{m}\left(1-1 / p_{i}\right)}}{\nu_{\vec{\omega}}\left(2^{j+1} B\right)^{1 / p}} \prod_{i=1}^{m}\left(\left\|f_{i}\right\|_{\mathscr{L}_{i}, k\left(w_{i}\right)} \omega_{i}\left(2^{j+1} B\right)^{k / p_{i}}\right) \\
& \leq C \prod_{i=1}^{m}\left\|f_{i}\right\|_{\mathscr{L}^{p_{i}, k}\left(w_{i}\right)} \sum_{j=1}^{\infty}\left(\frac{\prod_{i=1}^{m} \omega_{i}\left(2^{j+1} B\right)^{k / p_{i}}}{\nu_{\vec{\omega}}\left(2^{j+1} B\right)^{1 / p}}\right) \leq C \prod_{i=1}^{m}\left\|f_{i}\right\|_{\mathscr{L}^{p_{i}, k}\left(w_{i}\right)} \sum_{j=1}^{\infty} \nu_{\vec{\omega}}\left(2^{j+1} B\right)^{(k-1) / p} .
\end{aligned}
$$


Then, Lemma 19 implies that

$$
\begin{aligned}
I_{\vec{\alpha}} & \leq C \nu_{\vec{\omega}}(B)^{(1-k) / p}\left|T^{*}\left(f_{1}^{\infty}, \ldots, f_{m}^{\infty}\right)(x)\right| \\
& \leq C \prod_{i=1}^{m}\left\|f_{i}\right\|_{\mathscr{L}^{p_{i}, k}\left(w_{i}\right)} \sum_{j=1}^{\infty} \frac{v_{\vec{\omega}}(B)^{(1-k) / p}}{v_{\vec{\omega}}\left(2^{j+1} B\right)^{(1-k) / p}} \\
& \leq C \prod_{i=1}^{m}\left\|f_{i}\right\|_{\mathscr{L}^{p_{i}, k}\left(w_{i}\right)} \sum_{j=1}^{\infty}\left(\frac{|B|}{\left|2^{j+1} B\right|}\right)^{\delta(1-k) / p} \\
& \leq C \prod_{i=1}^{m}\left\|f_{i}\right\|_{\mathscr{L}^{p_{i}, k}\left(w_{i}\right)} .
\end{aligned}
$$

It remains for us to consider $I_{\vec{\alpha}}$ with $\vec{\alpha}=\left(\alpha_{1}, \ldots, \alpha_{m}\right), \alpha_{i}=0$ or $\infty$ for $i=1, \ldots, m$. We may assume that $\alpha_{1}=\cdots=\alpha_{l}=\infty$ and $\alpha_{l+1}=\cdots=\alpha_{m}=0$. Minkowski's inequality and the size condition (7) imply that

$$
\begin{aligned}
& \left|T^{*}\left(f_{1}^{\infty}, \ldots, f_{l}^{\infty}, f_{l+1}^{0}, \ldots, f_{m}^{0}\right)(x)\right| \\
& \quad \leq C \int_{\mathbb{R}^{n m}}\left(\int_{0}^{\infty}\left|K_{t}(x, \vec{y})\right|^{2} \frac{d t}{t}\right)^{1 / 2} \\
& \cdot \prod_{i=1}^{l} f_{i}^{\infty}\left(y_{i}\right) \prod_{i=l+1}^{m} f_{i}^{0}\left(y_{i}\right) d \vec{y} \\
& \quad \leq C \int_{\left(\mathbb{R}^{n} \backslash 2 B\right)^{l}} \int_{(2 B)^{m-l}} \frac{\prod_{i=1}^{l} f_{i}^{\infty}\left(y_{i}\right) \prod_{i=l+1}^{m} f_{i}^{0}\left(y_{i}\right)}{\left(\sum_{i=1}^{m}\left|x-y_{i}\right|\right)^{m n}} d \vec{y} \\
& \quad \leq C \prod_{i=l+1}^{m} \int_{2 B}\left|f_{i}\left(y_{i}\right)\right| d y_{i} \\
& \quad \sum_{j=1}^{\infty} \frac{1}{\left|2^{j+1} B\right|^{m}} \int_{\left(2^{j+1} B \backslash 2^{j} B\right)^{l}} \prod_{i=1}^{l} f_{i}\left(y_{i}\right) d y_{1} \cdots d y_{l} \\
& \quad \leq C \sum_{j=1}^{\infty} \prod_{i=1}^{m} \frac{1}{\left|2^{j+1} B\right|} \int_{2^{j+1} B}\left|f_{i}\left(y_{i}\right)\right| d y_{i} \\
& \leq C \prod_{i=1}^{m}\left\|f_{i}\right\|_{\mathscr{L}^{p_{i}, k}\left(w_{i}\right)} \sum_{j=1}^{\infty} \nu_{\vec{\omega}}\left(2^{j+1} B\right)^{(k-1) / p} .
\end{aligned}
$$

Together with Lemma 19, we obtain

$I_{\vec{\alpha}}$

$$
\begin{aligned}
& \leq C v_{\vec{\omega}}(B)^{(1-k) / p}\left|T^{*}\left(f_{1}^{\infty}, \ldots, f_{l}^{\infty}, f_{l+1}^{0}, \ldots, f_{m}^{0}\right)(x)\right| \\
& \leq C \prod_{i=1}^{m}\left\|f_{i}\right\|_{\mathscr{L}^{p_{i}, k}\left(w_{i}\right)} \sum_{j=1}^{\infty} \frac{\nu_{\vec{\omega}}(B)^{(1-k) / p}}{\nu_{\vec{\omega}}\left(2^{j+1} B\right)^{(1-k) / p}} \\
& \leq C \prod_{i=1}^{m}\left\|f_{i}\right\|_{\mathscr{L}^{p_{i}, k}\left(w_{i}\right)} \sum_{j=1}^{\infty}\left(\frac{|B|}{\left|2^{j+1} B\right|}\right)^{\delta(1-k) / p} \\
& \leq C \prod_{i=1}^{m}\left\|f_{i}\right\|_{\mathscr{L}^{p_{i}, k}\left(w_{i}\right)} .
\end{aligned}
$$

Combining the above estimates and then taking the supremum over all balls $B \in \mathbb{R}^{n}$, we complete the proof of Theorem 15(i).

We are now in a position to demonstrate (ii). For any $\lambda>$ 0 , we have

$$
\begin{aligned}
& \nu_{\vec{\omega}}\left(\left\{x \in B:\left|T^{*}\left(f_{1}, \ldots, f_{m}\right)(x)\right|>\lambda\right\}\right)^{1 / p} \leq C \nu_{\vec{\omega}}(\{x \\
& \left.\left.\quad \in B:\left|T^{*}\left(f_{1}^{0}, \ldots, f_{m}^{0}\right)(x)\right|>\lambda\right\}\right)^{1 / p} \\
& +C \sum_{\vec{\alpha}, \vec{\alpha} \neq \overrightarrow{0}} \nu_{\vec{\omega}}\left(\left\{x \in B:\left|T^{*}\left(f_{1}^{\alpha_{1}}, \ldots, f_{m}^{\alpha_{m}}\right)(x)\right|\right.\right. \\
& >\lambda\})^{1 / p}=C\left(P_{\overrightarrow{0}}+\sum_{\vec{\alpha}, \vec{\alpha} \neq \overrightarrow{0}} P_{\vec{\alpha}}\right) .
\end{aligned}
$$

Using Theorem 6(ii) and Lemmas 18 and 17, we obtain

$$
\begin{aligned}
P_{\overrightarrow{0}} & =\nu_{\vec{\omega}}\left(\left\{x \in B:\left|T^{*}\left(f_{1}^{0}, \ldots, f_{m}^{0}\right)(x)\right|>\lambda\right\}\right)^{1 / p} \\
& \leq \frac{C \prod_{i=1}^{m}\left(\int_{2 B}\left|f_{i}(x)\right|^{p_{i}} \omega_{i}(x) d x\right)^{1 / p_{i}}}{\lambda} \\
& \leq \frac{C \prod_{i=1}^{m} \omega_{i}(2 B)^{k / p_{i}}\left\|f_{i}\right\|_{\mathscr{L} p_{i}, k}\left(w_{i}\right)}{\lambda} \\
& \leq \frac{C \nu_{\vec{\omega}}(2 B)^{k / p} \prod_{i=1}^{m}\left\|f_{i}\right\|_{\mathscr{L}^{p_{i}, k}\left(w_{i}\right)}}{\lambda} \\
& \leq \frac{C \nu_{\vec{\omega}}(B)^{k / p} \prod_{i=1}^{m}\left\|f_{i}\right\|_{\mathscr{L}^{p_{i}, k}\left(w_{i}\right)}}{\lambda}
\end{aligned}
$$

We assume that $p_{1}=\cdots=p_{l}=1$ and $p_{l+1}, \ldots, p_{m}>1$. If $\vec{\alpha} \neq$ $\overrightarrow{0}$, recall that in $(60)$ and $(62)$ we have proved the following fact:

$$
\begin{aligned}
& \left|T^{*}\left(f_{1}^{\alpha_{1}}, \ldots, f_{m}^{\alpha_{m}}\right)(x)\right| \\
& \quad \leq C \sum_{j=1}^{\infty} \prod_{i=1}^{m} \frac{1}{\left|2^{j+1} B\right|} \int_{2^{j+1} B}\left|f_{i}\left(y_{i}\right)\right| d y_{i} .
\end{aligned}
$$

Hence, we may obtain

$$
\begin{aligned}
& \left|T^{*}\left(f_{1}^{\alpha_{1}}, \ldots, f_{m}^{\alpha_{m}}\right)(x)\right| \\
& \quad \leq C \sum_{j=1}^{\infty} \prod_{i=1}^{l} \frac{1}{\left|2^{j+1} B\right|} \int_{2^{j+1} B}\left|f_{i}\left(y_{i}\right)\right| d y_{i} \\
& \quad \cdot \prod_{i=l+1}^{m} \frac{1}{\left|2^{j+1} B\right|} \int_{2^{j+1} B}\left|f_{i}\left(y_{i}\right)\right| d y_{i} \\
& \quad \leq C \sum_{j=1}^{\infty} \prod_{i=1}^{l} \frac{1}{\left|2^{j+1} B\right|} \int_{2^{j+1} B}\left|f_{i}\left(y_{i}\right)\right| \omega_{i}\left(y_{i}\right) d y_{i} \\
& \quad \cdot\left(\inf _{y_{i} \in 2^{j+1} B} \omega_{i}\left(y_{i}\right)\right)^{-1} \\
& \quad \prod_{i=l+1}^{m} \frac{1}{\left|2^{j+1} B\right|}\left(\int_{2^{j+1} B}\left|f_{i}\left(y_{i}\right)\right|^{p_{i}} \omega_{i}\left(y_{i}\right) d y_{i}\right)^{1 / p_{i}}
\end{aligned}
$$




$$
\begin{aligned}
& \cdot\left(\int_{2^{j+1} B} \omega_{i}\left(y_{i}\right)^{1-p_{i}^{\prime}} d y_{i}\right)^{1 / p_{i}^{\prime}} \leq C \prod_{i=1}^{m}\left\|f_{i}\right\|_{\mathscr{L} p_{i}, k}\left(w_{i}\right) \\
& \cdot \sum_{j=1}^{\infty} \nu_{\vec{\omega}}\left(2^{j+1} B\right)^{(k-1) / p} \leq C \prod_{i=1}^{m}\left\|f_{i}\right\|_{\mathscr{L}^{p_{i}, k}\left(w_{i}\right)} \\
& \cdot \frac{1}{\nu_{\vec{\omega}}(B)^{(1-k) / p}} \cdot \sum_{j=1}^{\infty} \frac{\nu_{\vec{\omega}}(B)^{(1-k) / p}}{\nu_{\vec{\omega}}\left(2^{j+1} B\right)^{(k-1) / p}} \\
& \leq C \prod_{i=1}^{m}\left\|f_{i}\right\|_{\mathscr{L}^{p_{i}, k}\left(w_{i}\right)} \cdot \frac{1}{\nu_{\vec{\omega}}(B)^{(1-k) / p}} \\
& \cdot \sum_{j=1}^{\infty}\left(\frac{|B|}{\left|2^{j+1} B\right|}\right)^{\delta(1-k) / p} \leq C \prod_{i=1}^{m}\left\|f_{i}\right\|_{\mathscr{L}^{p_{i}, k}\left(w_{i}\right)} \\
& \cdot \frac{1}{\nu_{\vec{\omega}}(B)^{(1-k) / p}} \cdot
\end{aligned}
$$

To prove Theorem 15(ii), we may assume that

$$
\nu_{\vec{\omega}}\left(\left\{x \in B:\left|T^{*}\left(f_{1}^{\alpha_{1}}, \ldots, f_{m}^{\alpha_{m}}\right)(x)\right|>\lambda\right\}\right)>0 .
$$

Otherwise, there is nothing needing to be proved.

Then, by the above estimates, we obtain that

$$
\begin{gathered}
\nu_{\vec{\omega}}\left(\left\{x \in B:\left|T^{*}\left(f_{1}^{\alpha_{1}}, \ldots, f_{m}^{\alpha_{m}}\right)(x)\right|>\lambda\right\}\right) \\
\leq \frac{C \nu_{\vec{\omega}}(B) \prod_{i=1}^{m}\left\|f_{i}\right\|_{\mathscr{L}^{p_{i}, k}\left(w_{i}\right)}^{p}}{\lambda^{p} \cdot \nu_{\vec{\omega}}(B)^{1-k}} .
\end{gathered}
$$

Therefore, we get

$$
\begin{aligned}
& \frac{1}{\nu_{\vec{\omega}}(B)^{k / p}} \cdot \lambda \\
& \quad \cdot \nu_{\vec{\omega}}\left(\left\{x \in B:\left|T^{*}\left(f_{1}^{\alpha_{1}}, \ldots, f_{m}^{\alpha_{m}}\right)(x)\right|>\lambda\right\}\right)^{1 / p} \\
& \quad \leq C \prod_{i=1}^{m}\left\|f_{i}\right\|_{\mathscr{L}_{i}, k\left(w_{i}\right)} .
\end{aligned}
$$

This completes the proof of Theorem 15.

\section{Competing Interests}

The authors declare that they have no competing interests.

\section{Acknowledgments}

The first author was supported by the National Natural Science Foundation of China (no. 11401175 and no. 11501169). The second author was supported partly by NSFC (no. 11471041), the Fundamental Research Funds for the Central Universities (no. 2014kJJCA10), and NCET-13-0065.

\section{References}

[1] R. R. Coifman and Y. Meyer, Au-Delà des Opérateurs PseudoDifferentiels, Astérisque 57, Société Mathématique de France, Paris, France, 1978.
[2] K. Yabuta, "A multilinearization of Littlewood-Paley's $g$ function and Carleson measures," Tohoku Mathematical Journal, vol. 34, no. 2, pp. 251-275, 1982.

[3] S. Sato and K. Yabuta, "Multilinearized Littlewood-Paley operators," Scientiae Mathematicae Japonicae, vol. 55, no. 3, pp. 447453, 2002.

[4] Q. Xue, X. Peng, and K. Yabuta, "On the theory of multilinear Littlewood-Paley $g$-function," Journal of the Mathematical Society of Japan, vol. 67, no. 2, pp. 535-559, 2015.

[5] R. R. Coifman, D. Deng, and Y. Meyer, "Domaine de la racine carrée de certains opérateurs différentiels accrétifs," Annales de L'institut Fourier, vol. 33, no. 2, pp. 123-134, 1983.

[6] R. R. Coifman, A. McIntosh, and Y. Meyer, "L'integrale de Cauchy definit un operateur borne sur $L^{2}$ pour les courbes lipschitziennes," Annals of Mathematics, vol. 116, no. 2, pp. 361-387, 1982.

[7] G. David and J. L. Journe, "Une caractrisation des oprateurs intgraux singuliers borns sur $L^{2}\left(\mathbb{R}^{n}\right)$," Comptes Rendus de l'Académie des Sciences, vol. 296, pp. 761-764, 1983.

[8] E. B. Fabes, D. S. Jerison, and C. E. Kenig, "Multilinear Littlewood-Paley estimates with applications to partial differential equations," Proceedings of the National Academy of Sciences of the United States of America, vol. 79, no. 18, pp. 5746-5750, 1982.

[9] E. B. Fabes, D. S. Jerison, and C. E. Kenig, "Necessary and sufficient conditions for absolute continuity of elliptic-harmonic measure," Annals of Mathematics. Second Series, vol. 119, no. 1, pp. 121-141, 1984.

[10] E. B. Fabes, D. S. Jerison, and C. E. Kenig, "Multilinear square functions and partial differential equations," American Journal of Mathematics, vol. 107, no. 6, pp. 1325-1368, 1985.

[11] X. Chen, Q. Xue, and K. Yabuta, "On multilinear LittlewoodPaley operators," Nonlinear Analysis: Theory, Methods \& Applications, vol. 115, pp. 25-40, 2015.

[12] S. Shi, Q. Xue, and K. Yabuta, "On the boundedness of multilinear Littlewood-Paley $g_{\lambda}^{*}$ function," Journal de Mathématiques Pures et Appliquées, vol. 101, no. 3, pp. 394-413, 2014.

[13] A. K. Lerner, S. Ombrosi, C. Pérez, R. H. Torres, and R. TrujilloGonzález, "New maximal functions and multiple weights for the multilinear Calderón-Zygmund theory," Advances in Mathematics, vol. 220, no. 4, pp. 1222-1264, 2009.

[14] C. Fefferman and E. M. Stein, "Some maximal inequalities," American Journal of Mathematics, vol. 93, pp. 107-115, 1971.

[15] Q. Xue and J. Yan, "On multilinear square function and its applications to multilinear Littlewood-Paley operators with nonconvolution type kernels," Journal of Mathematical Analysis and Applications, vol. 422, no. 2, pp. 1342-1362, 2015.

[16] D. Maldonado and V. Naibo, "Weighted norm inequalities for paraproducts and bilinear pseudodifferential operators with mild regularity," The Journal of Fourier Analysis and Applications, vol. 15, no. 2, pp. 218-261, 2009.

[17] G. Lu and P. Zhang, "Multilinear Calderón-Zygmund operators with kernels of Dini's type and applications," Nonlinear Analysis. Theory, Methodse Applications, vol. 107, pp. 92-117, 2014.

[18] X. Chen, "Weighted estimates for maximal operator of multilinear singular intergral," Bulletin of the Polish Academy of Sciences-Mathematics, vol. 58, no. 2, pp. 129-135, 2010.

[19] J. Duoandikoetxea, Fourier Analysis, vol. 29 of Graduate Studies in Mathematics, American Mathematical Society, Providence, RI, USA, 2001. 
[20] L. Grafakos and R. H. Torres, "Maximal operator and weighted norm inequalities for multilinear singular integrals," Indiana University Mathematics Journal, vol. 51, no. 5, pp. 1261-1276, 2002.

[21] C. B. Morrey, "On the solutions of quasi-linear elliptic partial differential equations," Transactions of the American Mathematical Society, vol. 43, no. 1, pp. 126-166, 1938.

[22] Y. Komori and S. Shirai, "Weighted Morrey spaces and a singular integral operator," Mathematische Nachrichten, vol. 282, no. 2, pp. 219-231, 2009.

[23] H. Wang and W. Yi, "Multilinear singular and fractional integral operators on weighted Morrey spaces," Journal of Function Spaces and Applications, vol. 2013, Article ID 735795, 11 pages, 2013.

[24] R. R. Coifman and Y. Meyer, "On commutators of singular integrals and bilinear singular integrals," Transactions of the American Mathematical Society, vol. 212, pp. 315-331, 1975.

[25] R. R. Coifman and Y. Meyer, "Commutateurs d'intégrales singulières et opérateurs multilinéaires," Annales de l'institut Fourier, vol. 28, no. 3, pp. 177-202, 1978.

[26] L. Grafakos and R. H. Torres, "Multilinear Calderón-Zygmund theory," Advances in Mathematics, vol. 165, no. 1, pp. 124-164, 2002.

[27] J. Garcia-Cuerva and J. L. Rubio de Francia, Weighted Norm Inequalities and Related Topics, North-Holland, Amsterdam, The Netherlands, 1985. 


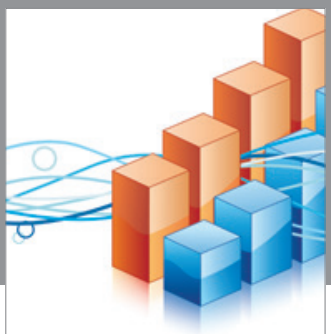

Advances in

Operations Research

vatem alat4

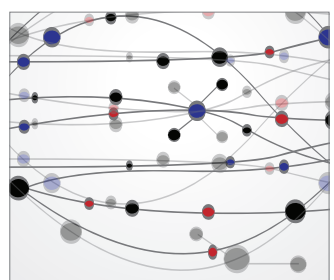

\section{The Scientific} World Journal
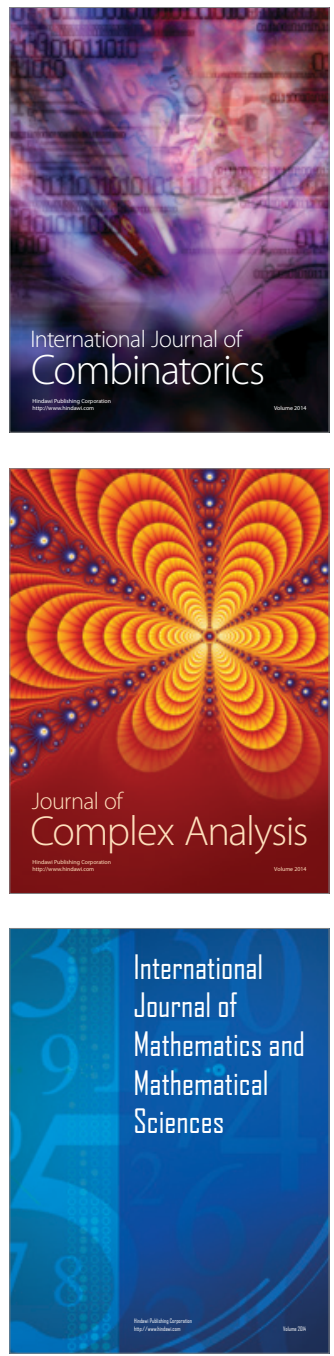
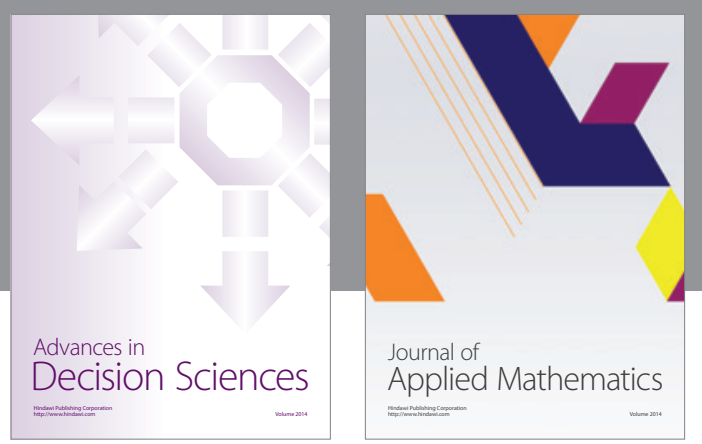

Algebra

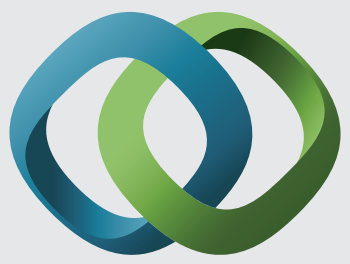

\section{Hindawi}

Submit your manuscripts at

http://www.hindawi.com
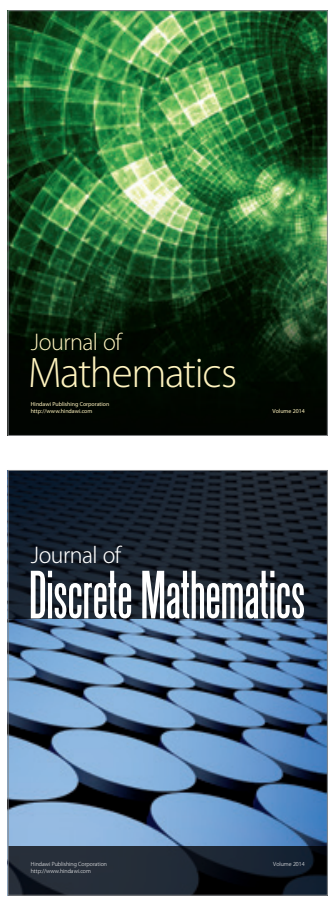

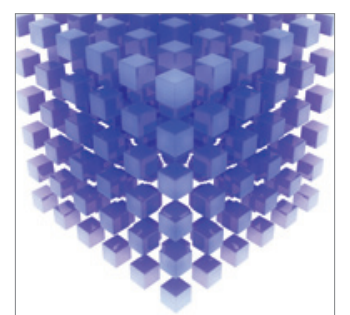

Mathematical Problems in Engineering
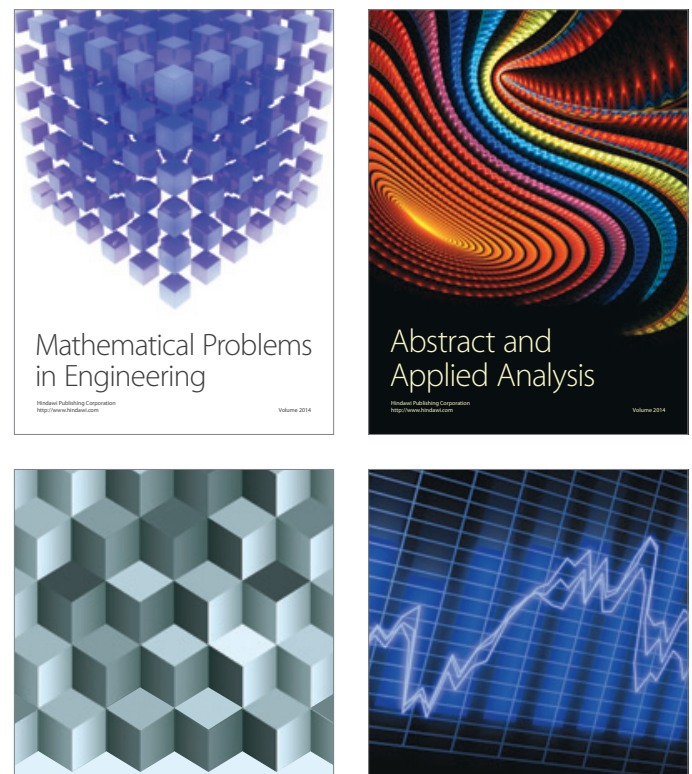

Journal of

Function Spaces

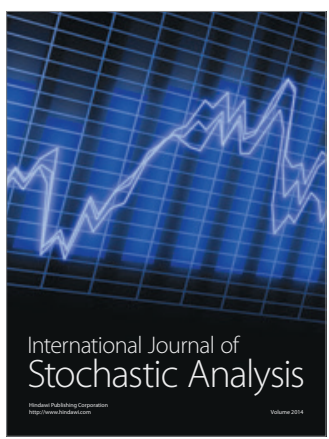

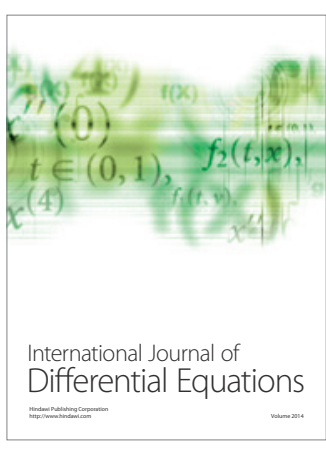
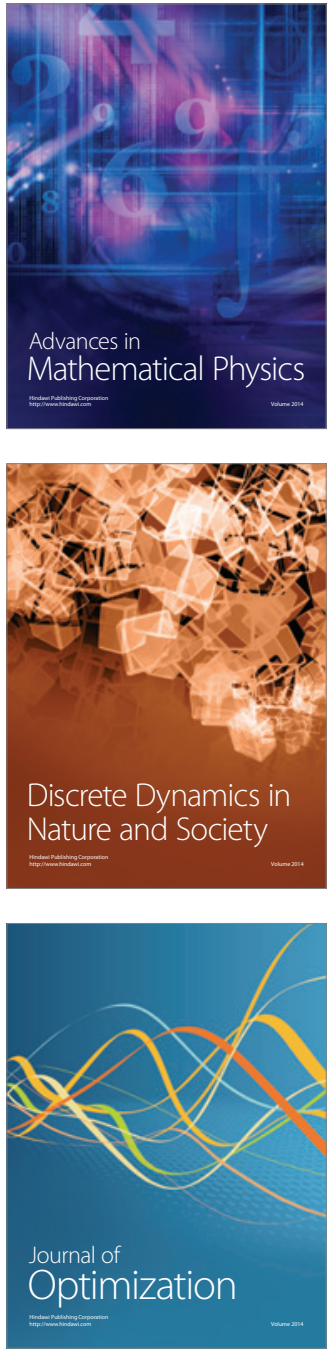